John Reinhold's valuable work on heart sounds and murmurs in children will be known to many readers. He had recently obtained a post as Assistant Professor of Padiatrics at Denver, Colorado, and was looking forward to his new opportunities. We deeply regret to record his death at the early age of 34, on July 19, 1957, as a result of injuries in a motor accident there.

\title{
RELATION OF THE FIRST AND SECOND HEART SOUNDS TO EVENTS IN THE CARDIAC CYCLE
}

\author{
BY \\ JOHN REINHOLD AND ULF RUDHE
}

From the Department of Child Health, Guy's Hospital, the Hospital for Sick Children, Great Ormond Street, London, and the Karolinska Sjukhuset, Stockholm

Received January 20, 1957

\begin{abstract}
"The chief value of the recorded heart sound is the possibility of accurately timing its occurrence in relation to the events of the cardiac cycle." This is even truer to-day than when Lewis first stated it (1915), because methods for recording events in the cardiac cycle have become more precise. In spite of this, there is still lack of agreement on the origin of the heart sounds. The causes of the second heart sound have been clearly defined, but there is a divergence of opinion on the origin of the major components of the first heart sound. On auscultation in young people the first sound may be heard to be narrowly split at the lower left sternal border. This splitting is widest and therefore most easily heard at the end of expiration (Potain, 1866). The origin of these two components has been attributed by some to closure of the auriculo-ventricular valves followed by opening of the semilunar valves (Orias and Braun-Menendez, 1939; Rappaport and Sprague, 1942; Luisada et al., 1949; Nazzi et al., 1954). Others have suggested that it is due to asynchronism in contraction of the ventricles (Wolferth and Margolies, 1945), and so to closure of the mitral valve followed by closure of the tricuspid valve (Leatham, 1954). Much of the evidence for these views has been obtained by relating the components of the heart sounds to dynamic events in the cardiac cycle by the use of reference tracings. Phlebograms and indirect carotid artery tracings have been mainly used for this purpose but these have the disadvantage that an arbitrary time interval has to be allowed for pulse wave transmission when assessing results.

The present investigation has been undertaken to study the nature of the first and second heart sounds and to relate their components to events in the cardiac cycle by the use of synchronous border electrokymograms. These tracings represent volume and positional changes of the heart chambers and the great vessels during the cardiac cycle. Positive waves indicate outward movement and negative waves indicate inward movement of the heart borders. These tracings have the advantage of eliminating the arbitrary time factor for pulse wave transmission.
\end{abstract}

\section{MethoDS}

In the first part of the investigation phonocardiograms were taken, using the Elmqvist sixchannel Klinik recorder, with three different frequency responses. These are designated high frequency, medium frequency, and low frequency according to the degree of attenuation of low frequency vibrations in each; the high and medium frequency tracings are similar to the logarithmic phonocardiogram of Rappaport and Sprague (1942) in their frequency response. Within the range of the heart sounds and murmurs the medium frequency channel has a pattern in its frequency response similar to the human audiogram; it thus represents the heart sounds as they are heard through the stethoscope. The low frequency tracings represent rather lower frequencies than are ordinarily heard through a stethoscope. Recordings were taken routinely from the apex, the fourth and second left intercostal spaces, and the second right intercostal space. Reference tracings 
were provided by lead II electrocardiograms, phlebograms, and carotid artery tracings recorded from the suprasternal notch; in some cases apex cardiograms also were recorded. Phlebograms and carotid tracings were obtained indirectly with a metal stylus resting over the vessel, impulses being transmitted directly to a crystal of Rochelle salt, and the voltage produced being amplified and subsequently recorded by a mirror galvanometer. It has been shown previously that there is no electrical delay in this circuit (Reinhold et al., 1955).

In the major part of this investigation medium frequency phonocardiograms were taken synchronously with border electrokymograms, lead II electrocardiograms and apex-cardiograms. Phonocardiograms were recorded from the four standard sites. Electrokymograms were taken from the base of the ascending aorta approximately $4-5 \mathrm{~cm}$. above the aortic valve, from the pulmonary artery close to the pulmonary valve, from the left and right auricles, and in many cases from the apices of the left and right ventricles. Recordings were also made from the aortic arch in a few patients. The majority of recordings were taken with the patient in the erect position. The electrical delay in the electrokymogram circuit compared with that of the phonocardiogram was slightly less than $0.01 \mathrm{sec}$. This was estimated by feeding an electric signal to the inputs of the electrocardiogram, apex-cardiogram, phonocardiogram, and electrokymogram amplifiers. The response of each was recorded simultaneously and showed no time lag between the electrocardiogram, apex-cardiogram and phonocardiogram, and slightly less than $0.01 \mathrm{sec}$. lag of the electrokymogram behind these three tracings. Consequently, an allowance of $0.01 \mathrm{sec}$. has been made in all tracings for this delay.

\section{INTERPRETATION OF THE NORMAL ELECTROKYMOGRAM}

Aotric valve opening was denoted by the point of upstroke of the electrokymogram recorded from the ascending aorta, no time allowance being necessary for pulse wave transmission. In some instances there was a small positive wave (Fig. 1A) or an inflexion (Fig. 1B) preceding the main

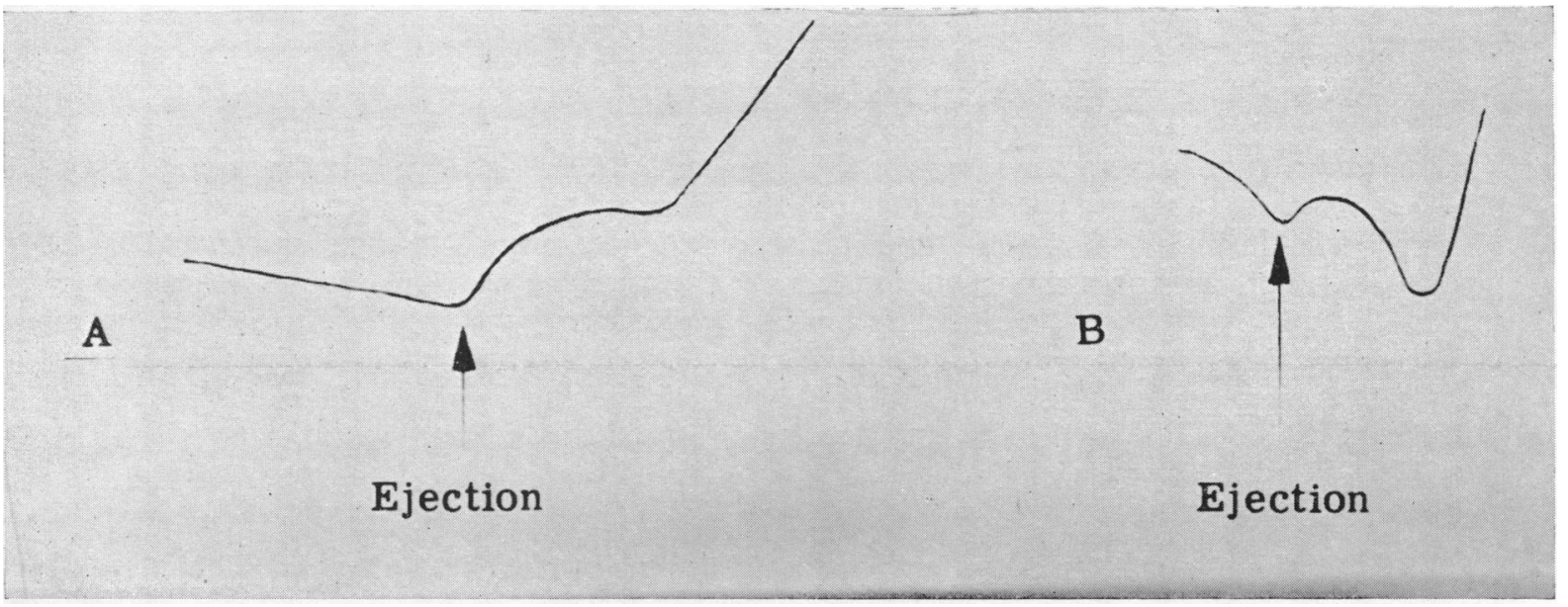

FIG. 1.-Electrokymogram from ascending aorta (see text).

upstroke. By comparison with left ventricular and aortic arch tracings it was apparent that the onset of ejection into the aorta was denoted by the first rise on these tracings $(\uparrow)$. The cause for this distortion of the ejection wave is probably the brisk downward movement of the base of the heart that occurs at the beginning of the isotonic phase of ventricular systole (Luisada, 1953). Aotric valve closure was denoted by the dicrotic notch on the same tracing.

Pulmonary valve opening was denoted by the point of upstroke of the pulmonary artery electro- 
kymogram, no time allowance for pulse wave transmission being necessary because the tracings were recorded from the artery immediately beyond the valve. Pulmonary valve closure was denoted by the dicrotic notch on this electrokymogram.

The point that was considered to be synchronous with mitral valve closure was determined from a typical inflexion on the left auricular electrokymogram. It has been stated by Braunwald et al. (1955) that mitral valve closure is virtually simultaneous with the onset of isometric left ventricular contraction. The end of auricular contraction $\left(\mathrm{A}_{2}\right)$ and the beginning of the isometric ventricular phase on the left auricular electrokymogram $\left(S_{1}\right)$ are often simultaneous and are marked by the onset of a positive wave following the negative auricular systolic wave (Mednick et al., 1950; Rudhe, 1956). This point has been taken as indicating the time of mitral valve closure. On the right auricular electrokymogram, there is often a brief interval between the end of auricular systole and the onset of isometric right ventricular contraction. This is usually denoted by a slight rise in gradient of the tracing after $A_{2}$ followed by a sharp inflexion $\left(S_{1}\right)$, which is in turn succeeded by a steeper rise to $S_{2}$ (Fig. 2). Although $S_{1}$ is not always present on this tracing, we have confined our observations to those records in which this inflexion is clearly visible.

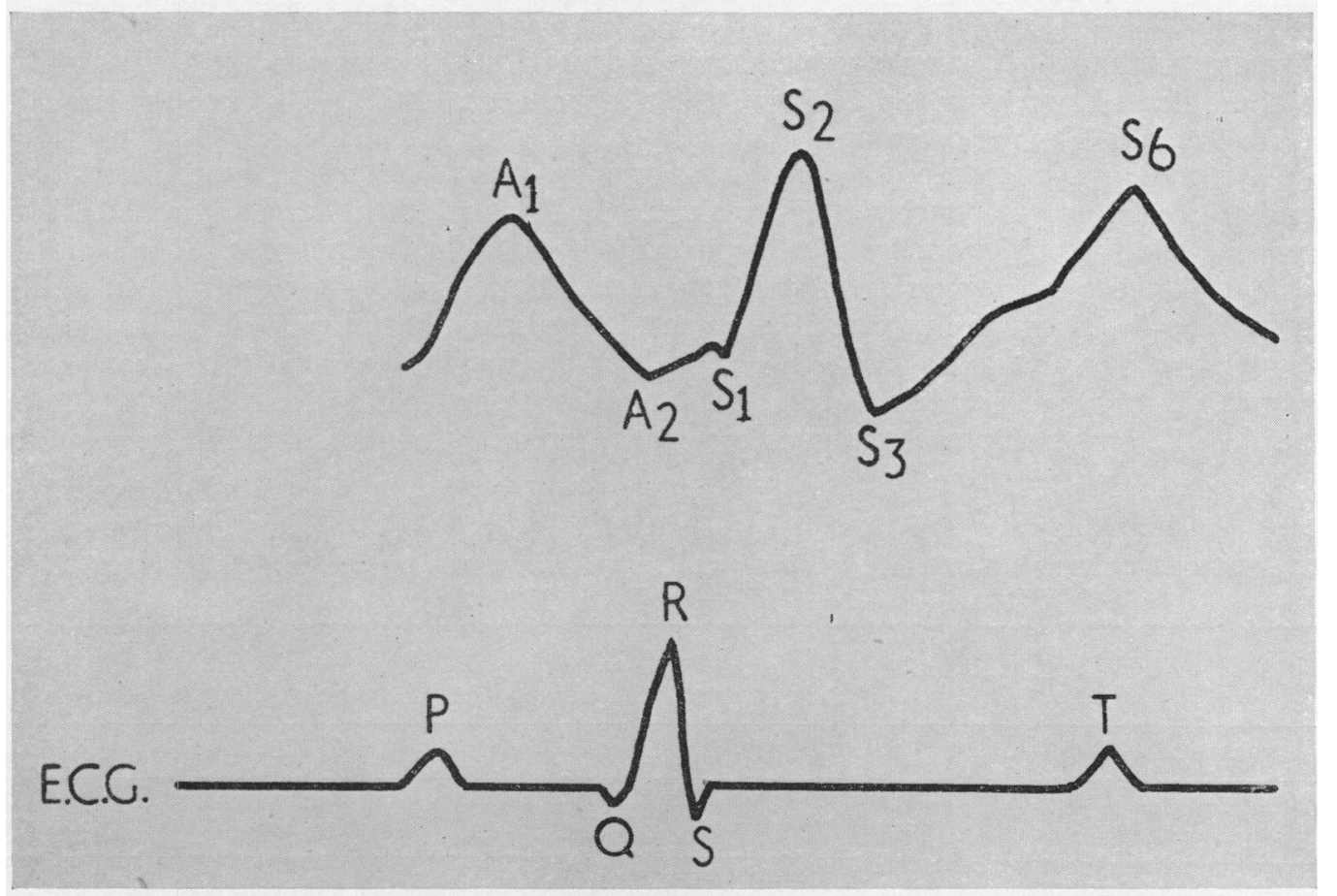

Fig. 2.-Electrokymogram from right auricle.

It is generally believed that this point denotes the onset of isometric right ventricular systole (Booth et al., 1953; Rudhe, 1956), and is therefore synchronous with closure of the tricuspid valve These points of reference have been checked by comparison with right and left ventricular electrokymograms where the onset of isometric contraction is denoted by a preliminary downward inflexion before the main ejection wave (Mednick et al., 1950).

It is generally agreed that opening of the mitral and tricuspid valves is marked by the point where the auricular diastolic wave ends $\left(S_{6}\right)$ and where there is a sharp fall in the curve with the onset of the first rapid phase of ventricular filling. It has been shown that this point corresponds 
closely to the fall in pressure on the right auricular pressure curve at the end of auricular diastole (Rudhe, 1956).

\section{MATERIAL}

Forty-five normal children and young adults were included in the investigation. Twenty were investigated with the aid of electrokymograms but in only sixteen were the points on the auricular tracings defined clearly enough for analysis of their relationship to the first heart sound. The remaining subjects were examined with carotid or jugular reference tracings. One patient with complete heart block was included to study the relationship of the time of auricular systole to the amplitude of the components of the first heart sound.

\section{RESULTS}

In many subjects, careful auscultation revealed splitting of the first heart sound, best heard at the lower left sternal border. In all subjects splitting of the second heart sound was heard at the pulmonary area.

\section{The First Sound}

In many previous studies the first sound has been divided into four components (Orias and BraunMenendez, 1939; Rappaport and Sprague, 1942; Nazzi et al., 1954). Of these the second and third are the audible major components.

In 33 normal subjects studied by phonocardiography it has been possible to separate the first sound into these four components in all instances. In this work these have been called the initial component, the first and second major components and the final component. The major components, which give the impression of splitting on auscultation, were best recorded at the lower left sternal border although they were also often well registered at the apex. The duration of the first sound and of its individual components has been measured in each subject on the medium frequency tracing at the apex, and the site of maximum registration of each component has been recorded (Table I). We have made no attempt to record the number of vibrations making up each com-

TABLE I

The Average Duration and the Site of Maximum Registration of the 1st Sound in 33 Normal Subjects (Medium Frequency Channel)

\begin{tabular}{|c|c|c|c|c|c|c|c|}
\hline & & & & $\begin{array}{c}\text { Initial } \\
\text { component }\end{array}$ & $\begin{array}{l}\text { 1st major } \\
\text { component }\end{array}$ & $\begin{array}{l}\text { 2nd major } \\
\text { component }\end{array}$ & $\begin{array}{c}\text { Final } \\
\text { component }\end{array}$ \\
\hline Site of maximun & n registration & .. & .. & Apex & Apex & 4. L.I.S. & Base \\
\hline $\begin{array}{l}\text { Duration (sec.) } \\
\text { (Range) } \quad .\end{array}$ & $\begin{array}{ll}. & . \\
. . & .\end{array}$ & $\begin{array}{l}. . \\
\cdots\end{array}$ & 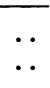 & $\begin{array}{c}0.04 \\
(0.02-0.05)\end{array}$ & $\begin{array}{c}0.03 \\
(0.02-0.04)\end{array}$ & $\begin{array}{c}0.02 \\
(0.01-0.04)\end{array}$ & $\begin{array}{c}0.04 \\
(0.01-0.07)\end{array}$ \\
\hline Total duration 0 & f 1st sound. & (sec.) & .. & \multicolumn{4}{|c|}{$0.13(0.09-0.15)$} \\
\hline
\end{tabular}

ponent as this varies with the method of recording (Mannheimer, 1955). Neither have we attempted to measure the frequency of each component because the first heart sound, being composed of a conglomeration of unrelated frequencies, cannot be regarded as a pure harmonic vibration. Moreover, the vibrational frequencies of the heart sounds vary with the frequency response of the apparatus and the type of chest piece employed (Rappaport and Sprague, 1942). However, we have attempted to determine whether one component contained more higher frequency vibrations than another in the medium frequency channel.

The initial component was of lower amplitude than the first and second major components in all areas and was best registered at the apex. In the majority of subjects it was not registered over the 
base of the heart. It appeared to be mainly composed of low frequency vibrations. By means of the synchronous left auricular electrokymogram it was shown that this component occurred in the pre-isometric phase immediately before the onset of isometric left ventricular contraction.

The first major component was registered in all areas, but best at the apex. It was of considerably greater amplitude than the initial component and, in many subjects, it was of slightly greater amplitude than the second major component at the apex. Both major components appeared to contain more high frequency vibrations than the initial and final components.

By comparison with the left auricular electrokymogram (Fig. 3), the onset of the first major component was correlated with the onset of left ventricular isometric contraction and the time of mitral valve closure (Table II). The two events were synchronous in 11 out of 16 instances, and

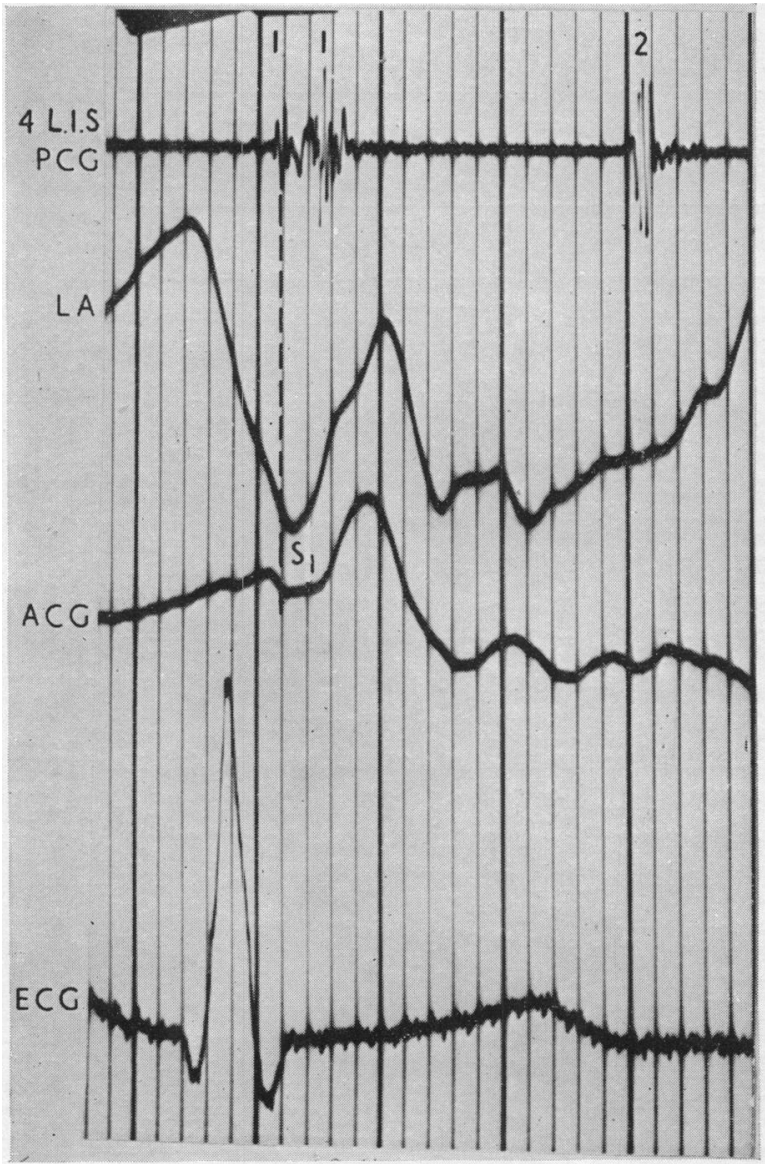

Fig. 3.-Synchronous medium frequency phonocardiogram (PCG) and left auricular electrokymogram (LA) in a normal child, showing relationship of onset of left ventricular isometric contraction $\left(\mathbf{S}_{1}\right)$ to the first major component of the first sound. (Enlarged $\times 2$.) 4 L.I.S. = Fourth left intercostal space. $\mathrm{ACG}=$ apexcardiogram. ECG=electrocardiogram. Time intervals 0.02 and $0.1 \mathrm{sec}$. $0.01 \mathrm{sec}$. delay on electrokymogram has been allowed for in this and all subsequent tracings.

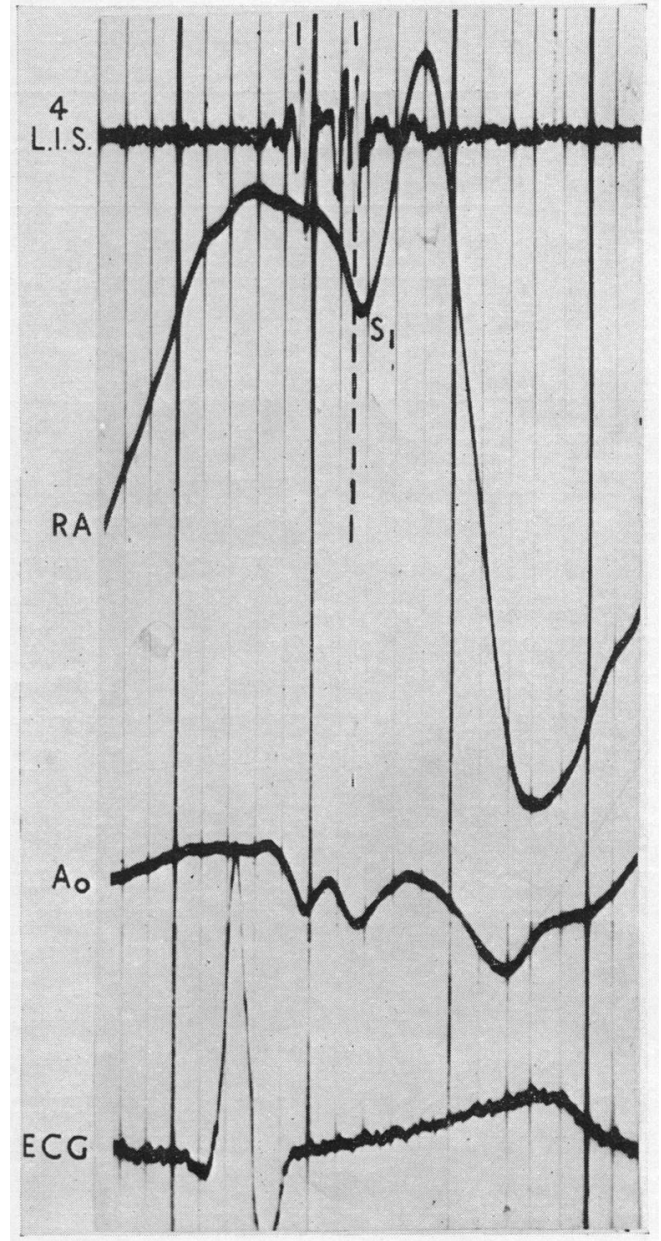

Fig. 4.- Synchronous medium frequency phonocardiogram and right auricular electrokymogram (RA) in a normal child, showing relationship of onset of right ventricular isometric contraction $\left(S_{1}\right)$ to the second major component of the first sound. (Enlarged $\times 2$.)

Note. Ao should be ACG, apex-cardiogram. 
TABLE II

The Relation of the Components of the 1st Sound to Events in the Cardiac Cycle in Normal Subjects DEFINED BY SYNCHRONOUS ELECTROKYMOGRAMS

\begin{tabular}{|c|c|c|c|c|c|}
\hline \multicolumn{4}{|c|}{ Interval } & \multirow{2}{*}{$\begin{array}{c}\begin{array}{c}\text { Average duration } \\
\text { in sec. (range) }\end{array} \\
0.04(0.02-0.05)\end{array}$} & \multirow{2}{*}{$\begin{array}{c}\begin{array}{c}\text { No. of } \\
\text { observations }\end{array} \\
17\end{array}$} \\
\hline Q-Initial component & .. & .. & .. & & \\
\hline Q-1st major component & .. & .. & .. & $0.07(0.05-0.09)$ & 16 \\
\hline $\begin{array}{c}\text { Q-Onset of L.V. isometri } \\
\text { closure) }\end{array}$ & ic ph & e. $(1$ & $\begin{array}{c}\text { Mitral valve } \\
\ldots \quad . .\end{array}$ & $0.08(0.05-0.09)$ & 16 \\
\hline Q-2nd major component & .. & . & . & $0.10(0.08-0.13)$ & 16 \\
\hline $\begin{array}{l}\text { Q-Onset of R.V. isome } \\
\text { valve closure) }\end{array}$ & tric & $\begin{array}{l}\text { hase. } \\
\ldots\end{array}$ & $\begin{array}{l}\text { (Tricuspid } \\
\ldots \quad \cdots\end{array}$ & $0 \cdot 10(0.07-0.13)$ & 16 \\
\hline Q-Final component & .. & . & .. & $0 \cdot 12(0 \cdot 10-0 \cdot 16)$ & 17 \\
\hline Q-Aortic valve opening & . & . & .. & $0 \cdot 11(0 \cdot 08-0 \cdot 15)$ & 17 \\
\hline Q-Pulmonary valve open & ing & .. & $\ldots$ & $0.13(0.07-0.17)$ & 17 \\
\hline
\end{tabular}

$0.01 \mathrm{sec}$. has been subtracted for electrical delay in the electrokymograph circuit.

the first major component preceded aortic and pulmonary valve opening by an average of 0.04 and 0.05 sec. respectively.

The second major component was registered in all areas, but best at the fourth left intercostal space, where, in most subjects, it was of greater amplitude than the first major component. It followed the first major component by an average interval of $0.03 \mathrm{sec}$.

By means of the right auricular electrokymogram, the second major component was shown to be synchronous with the notch on this tracing $\left(\mathrm{S}_{1}\right)$ indicating the onset of isometric right ventricular systole and tricuspid valve closure in 12 out of 16 instances (Fig. 4). The second major component preceded aortic (Fig. 5) and pulmonary valve opening (Fig. 6) in nearly all subjects (Table II).

The final component was registered in all areas but best over the base of the heart at the second left and second right intercostal spaces; in a few instances it was best recorded at the apex. These vibrations occurred at the time of or immediately after the opening of the aortic and pulmonary valves (Fig. 5 and 6), and were of lower amplitude than the major components and contained a preponderance of lower frequency vibrations.

\section{The Effect of Auricular Contraction on the Amplitude of the First Heart Sound}

It is known that the intensity of the first heart sound varies with the P-R interval, the sound being louder when the interval is short and softer when it is long (Griffith, 1911-12; Lewis, 1915). This is probably due to the effect of auricular contraction upon the position of the auriculo-ventricular valves. If the $\mathrm{P}-\mathrm{R}$ interval is long the valves have time to float upwards towards the position of closure before isometric ventricular systole begins and finally causes them to close. Therefore they will give rise to a soft sound on closing. If the P-R interval is short, the onset of the isometric phase occurs before auricular systole has ended and the auriculo-ventricular valves are still wide open and so come together with a loud snap (Wolferth and Margolies, 1930). In the light of this interpretation, if the two major components of the first sound are related to mitral and tricuspid valve closure, one might expect that they would vary in amplitude independently of each other and according to their individual time relationship with auricular systole. This phenomenon has indeed been demonstrated in two cases of complete heart block by Wolferth and Margolies 

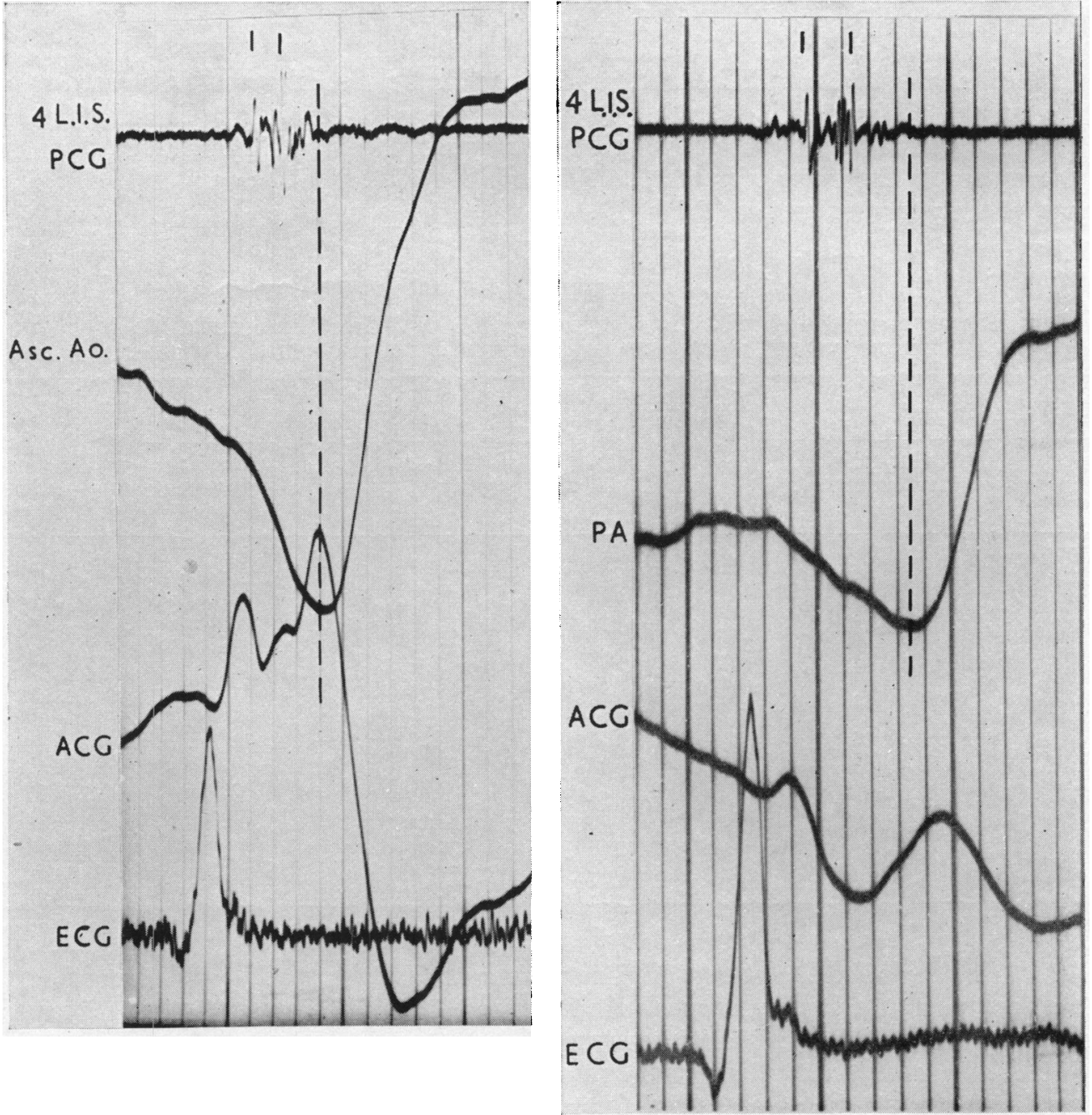

FIG. 5.-Synchronous medium frequency phonocardiogram and electrokymogram from base of ascending aorta (Asc. Ao.), showing that the major components of the first sound occur before aortic valve opening and the onset of systolic ejection into the aorta. (Enlarged $\times 2$.)

Fig. 6.-Synchronous medium frequency phonocardiogram and electrokymogram from the base of the pulmonary artery (PA), showing that the major components of the first sound occur before pulmonary valve opening and the onset of systolic ejection into the pulmonary artery. (Enlarged $\times 2$.)

(1935). They showed that each component of the first heart sound had its own clear-cut relationship to the $P$ wave by measuring the interval between the onset of $P$ and the individual components in each complex. They also found that the time zone of intervals between $P$ waves and sound components during which sound vibrations of large and small amplitude were recorded, differed very little for the two components. From this they deduced that the amplitude of the two components was dependent on the different auriculo-ventricular intervals on the two sides of the heart. They pointed out, however, that their investigation would have been greatly simplified if they had been able to measure the auriculo-ventricular intervals on the two sides by other than electrocardiographic methods.

With the aid of electrokymography we have had the opportunity of studying the relationship of 2K 
left and right auricular systole to the amplitude of the major components of the first heart sound in one patient with complete heart block. In Table III the time interval between the onset of left and right auricular systole and the first and second components of the first sound has been compared with their amplitude from cycle to cycle. Although the onset of auricular systole on the electrokymogram is later than that obtained by auricular pressure recordings (Rudhe, 1956), there appears to be a comparable time range for the two sides of the heart when the amplitude of the appropriate sound component is greater (Table III).

TABLE III

The Relationship of the P-R Interval and of the Time of Auricular Contraction, Measured on the Electrokymogram, to the Amplitude of the First HeARt Sound Components

\begin{tabular}{|c|c|c|c|c|c|}
\hline \multicolumn{2}{|c|}{ Time interval } & \multirow{2}{*}{$\begin{array}{l}\text { Max. amp. } \\
\text { of 1st } \\
\text { component } \\
\text { (mm.) }\end{array}$} & \multicolumn{2}{|c|}{ Time interval } & \multirow{2}{*}{$\begin{array}{l}\text { Max. amp. } \\
\text { of } 2 \text { nd } \\
\text { component } \\
\text { (mm.) }\end{array}$} \\
\hline $\begin{array}{l}\text { P-R } \\
\text { (sec.) }\end{array}$ & $\begin{array}{l}\mathrm{A}_{1} \text { (Lt. auricle) } \\
\text { to } 1 \text { st component } \\
\text { (sec.) }\end{array}$ & & $\begin{array}{c}\mathrm{P}-\mathrm{R} \text { or } \mathrm{R}-\mathrm{P} \\
\text { (sec.) }\end{array}$ & $\begin{array}{c}A_{1} \text { (Rt. auricle) } \\
\text { to 2nd component } \\
\text { (sec.) }\end{array}$ & \\
\hline $\begin{array}{l}0.02 \\
0.04 \\
0 \cdot 10 \\
0 \cdot 12 \\
0 \cdot 14 \\
0 \cdot 14 \\
0 \cdot 18 \\
0.21 \\
0.24 \\
0.30 \\
0.34\end{array}$ & $\begin{array}{l}0.01 \\
0.04 \\
0.06 \\
0.08 \\
0.08 \\
0.11 \\
0.15 \\
0.19 \\
0.21 \\
0.29 \\
0.30\end{array}$ & $\begin{array}{r}5 \\
8 \\
30 \\
24 \\
22 \\
19 \\
5 \\
3 \\
4 \\
5 \\
8\end{array}$ & $\begin{array}{l}\frac{\mathrm{R}-\mathrm{P}}{\mathbf{0} 07} \\
\frac{\mathrm{P}-\mathrm{R}}{0.06} \\
0.06 \\
0.08 \\
0.08 \\
0.11 \\
0.11 \\
0.13 \\
0.26 \\
0.32 \\
0.33\end{array}$ & $\begin{array}{l}\frac{2-\mathrm{A}_{1}}{0.06} \\
\frac{\mathrm{A}_{1}-2}{0.04} \\
0.05 \\
0.06 \\
0.08 \\
0.10 \\
0.10 \\
0.12 \\
0.24 \\
0.30 \\
0.32\end{array}$ & $\begin{array}{r}30 \\
34 \\
38 \\
36 \\
28 \\
26 \\
12 \\
4 \\
5 \\
8\end{array}$ \\
\hline
\end{tabular}

On the left side, when the auricle begins to contract (as estimated by the electrokymogram) $0.06-0.11 \mathrm{sec}$. before the first component, the latter is increased in amplitude; but earlier or later contraction of this auricle is associated with much lower amplitude of the first component. On the right side, when the onset of auricular contraction (similarly estimated) is $0.04-0.10 \mathrm{sec}$. before the second component, the amplitude of the sound is increased. Earlier or later contraction of the auricle is associated with lower amplitude of the second component. This relationship of auricular contraction and of the P-R interval to the amplitude of the first sound components is seen in Fig. 8. In beat 1 the auricles contract too early to affect either component; in beat 2 the auricles contract nearer to the first sound so that both components are affected, but the first is of greater amplitude than the second. In beat 3 both components are equally increased in amplitude by their relationship to auricular contraction and in subsequent beats the auricle is contracting too close to the first sound to affect the first component, but the second is accentuated. These findings confirm those of Wolferth and Margolies and support their postulate that the two major components of the first heart sound are due to similar events occurring asynchronously in opposite sides of the heart.

\section{The Second Sound}

For a long time it has been believed that the major components of the second heart sound are due to closure of the semilunar valves (Carswell, 1832; Billing, 1832; Rouanet, 1832). Splitting of the second sound in the pulmonary area is well recognized as a normal phenomenon, and it is known that this splitting increases with inspiration, and diminishes with expiration (Potain, 1866). The first component has been shown to be due to aortic valve closure by its relationship to the dicrotic 


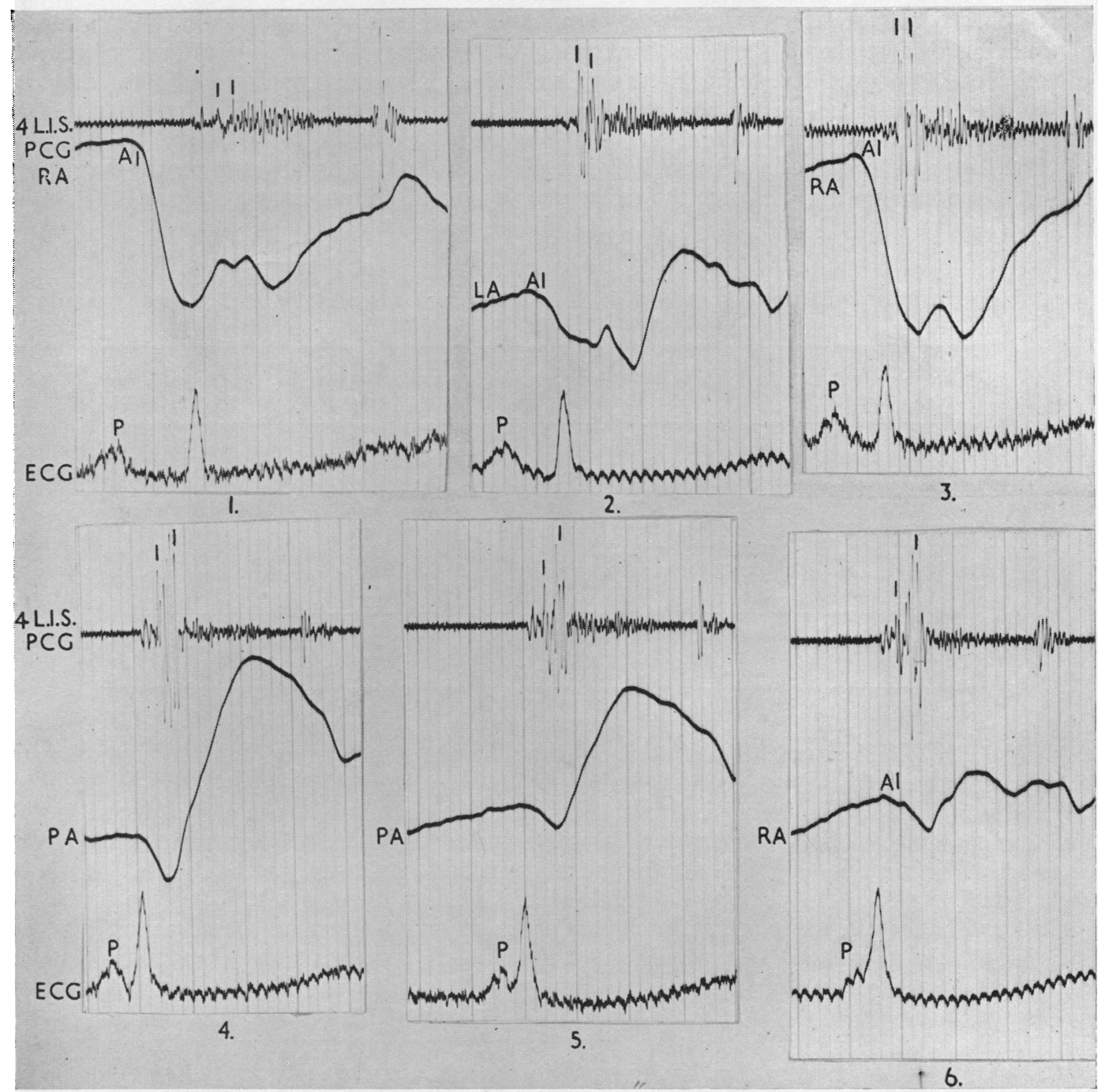

FIG. 7.-Synchronous medium frequency phonocardiograms, electrocardiograms, and electrokymograms from left auricle (LA), right auricle (RA) and pulmonary artery (PA) in a child with complete heart block. The individual tracings show the relationship between the amplitude of the major components of the first heart sound, the time of onset of auricular systole on the electrokymogram and the P-R interval.

notch of the carotid tracing and it alone is responsible for the second sound at the aortic area and the apex. The second component, being maximal at the second left intercostal space, has been thought to be due to pulmonary valve closure (Leatham, 1954). It has also been suggested that small low frequency vibrations following the major component at the apex are due to opening of the auriculoventricular valves (Rappaport and Sprague, 1942).

Analysis of the phonocardiogram in 45 normal young people showed the major component of the second sound to be single at the apex and second right intercostal spaces and split at the second left intercostal space in all instances. The second major component of the second sound was also recorded at the fourth left intercostal space, but with diminished amplitude. At the apex and 
the fourth left intercostal space, a few low amplitude vibrations of short duration were recorded after the major component; these were best seen on the low-frequency tracing. The characteristics of the second sound and its relationship to events in the cardiac cycle are shown in Table IV.

Splitting of the second sound varied with respiration and was greatest on inspiration, and often disappeared at the end of expiration.

The first major component was timed against the dicrotic notch on the electrokymogram taken from the ascending aorta, and was shown to precede it in all instances by an average interval of

\section{TABLE IV}

Characteristics of the Second Sound and its Relation to Events in the Cardiac Cycle by Reference TO SYNCHRONOUS ELECTROKYMOGRAMS

\begin{tabular}{|c|c|c|}
\hline Interval & $\begin{array}{l}\text { Average duration } \\
\text { in sec. (range) }\end{array}$ & $\begin{array}{l}\text { No. of } \\
\text { observations }\end{array}$ \\
\hline 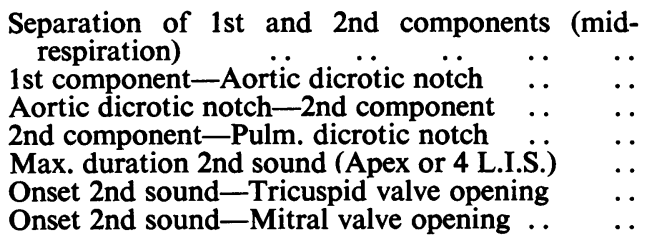 & $\begin{array}{l}0.05(0.03-0.07) \\
0.03(0.02-0.03) \\
0.02(0.01-0.05) \\
0.02(0.01-0.03) \\
0.08(0.06-0.10) \\
0.07(0.04-0.10) \\
0.10(0.04-0.14)\end{array}$ & $\begin{array}{l}45 \\
20 \\
21 \\
20 \\
21 \\
21 \\
20\end{array}$ \\
\hline $\begin{array}{l}\text { Comparative amplitude of } 1 \text { st to } 2 \text { nd component } \\
\text { at } 2 \text { L.I.S. } \ldots \\
. .\end{array}$ & $1 \cdot 5: 1(0 \cdot 9-5 \cdot 0: 1)$ & 37 \\
\hline
\end{tabular}

0.03 sec. (Fig. 8 ). It preceded the dicrotic notch on the pulmonary artery electrokymogram by an average of $0.07 \mathrm{sec}$.

The second major component occurred after the aortic dicrotic notch in all instances (Fig. 8) and thus could not be due to aortic valve closure. It preceded the dicrotic notch on the pulmonary artery electrokymogram (Fig. 9) indicating its relationship to pulmonary valve closure. It will be seen from Table IV that there was a short delay of the aortic dicrotic notch after the first component and of the pulmonary dicrotic notch after the second component. It seems unlikely that this delay is due to pulse wave transmission as the electrokymograms were taken from points on the aorta and pulmonary artery close to the semilunar valves; it is probable that it is accounted for by positional changes of the arteries at the time of valve closure (Rudhe, 1956).

The terminal low amplitude vibrations on the medium frequency tracing were related to opening of the mitral and tricuspid valves by reference to the left and right auricular electrokymograms (Table IV). The tricuspid valve opened shortly before the mitral valve in 17 instances and soon after the mitral valve in 2 instances; in one both valves opened simultaneously. Tricuspid valve opening was synchronous with the terminal vibrations of the second sound in 16 subjects and occurred after these vibrations in 5. Mitral valve opening was synchronous with these vibrations in 6 subjects and occurred after them in 14.

\section{The First Heart Sound}

\section{Discussion}

The vibrations that constitute the first heart sound have been attributed by different authors to muscular, vascular, and valvular events in the cardiac cycle. For some time, the initial vibrations were thought to be due to auricular factors (Bramwell, 1935; Cossio and Lascales, 1936; Orias and Braun-Menendez, 1939; Rappaport and Sprague, 1942); but it is now believed that the auricle plays no part in the production of the first sound (Wiggers and Dean, 1917; Lian et al., 1941; 


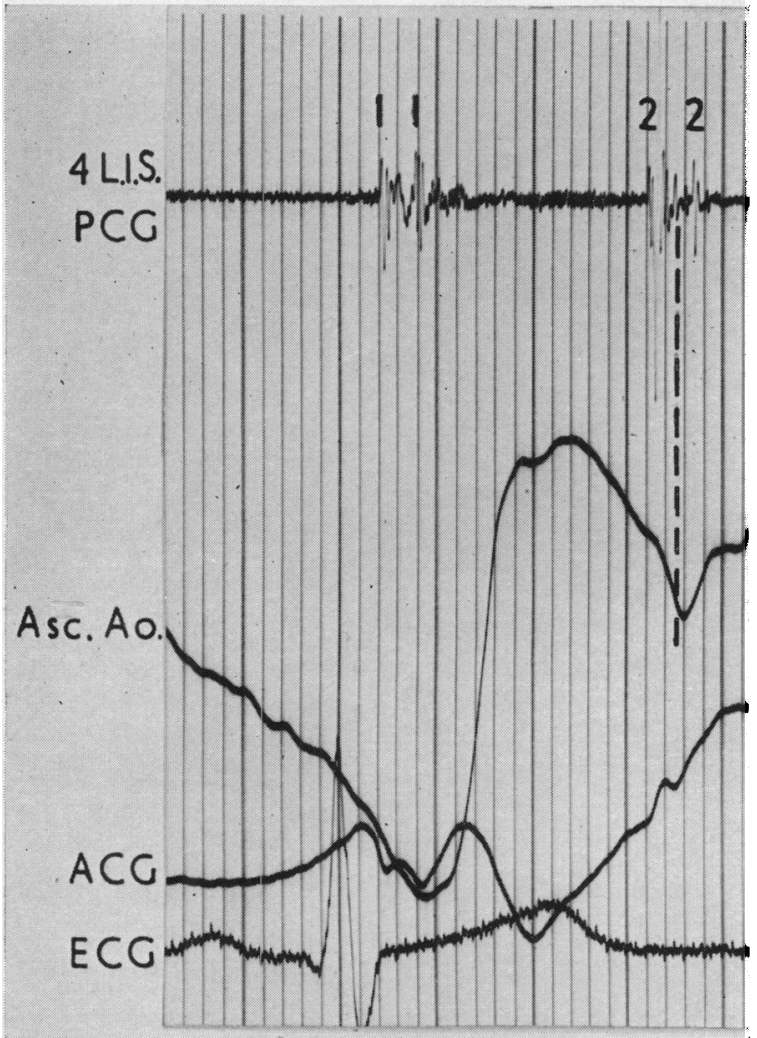

FIG. 8.-Synchronous medium frequency phonocardiogram and electrokymograms from the base of the ascending aorta (Asc. Ao.) in a normal child, showing the relationship of the aortic dicrotic notch to the major components of the split second sound.

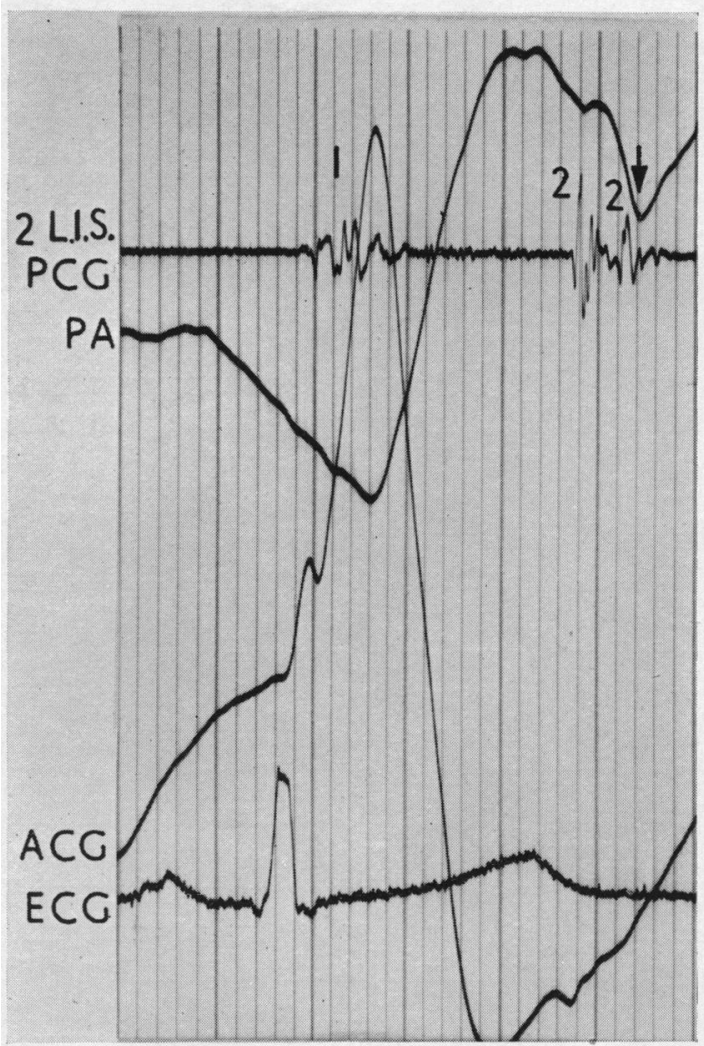

FIG. 9.-Synchronous medium frequency phonocardiograms and electrokymograms from the base of the pulmonary artery in a normal child, showing the relationship of the pulmonary dicrotic notch to the second major component of the split second sound. 2 L.I.S. = Second left intercostal space.

Counihan et al., 1951), and that the initial vibrations, occurring in the pre-isometric phase of ventricular systole, are caused by muscular factors due to changes in the shape of the ventricle immediately prior to the onset of isometric contraction (Nazzi et al., 1954).

There is general agreement that the onset of the first major component occurs approximately at the time of isometric ventricular contraction (Wiggers and Dean, 1917; Orias and BraunMenendez, 1939; Rappaport and Sprague, 1942; Wolferth and Margolies, 1945; Leatham and Vogelpoel, 1954; Nazzi et al., 1954). There is much less agreement on the relationship of the second major component to the isometric and the ejection phases of systole. Some have stated that this component is related to the onset of systolic ejection and is either due to vibration of the valve cusps before they open (Geigel, 1906), or to the actual opening of the valves (Einthoven and Geluk, 1894). Others believe that systolic ejection and semilunar valve opening do not contribute to the major components, and that auriculo-ventricular valve closure is mainly responsible for the production of the first heart sound (Rouanet, 1832; Dock, 1933). Recently Leatham (1954) has suggested that the major components of this sound are due to asynchronous mitral and tricuspid valve closure and he has pointed out that the first component is louder at the apex and the second at the lower left sternal border. Wolferth and Margolies (1945) support the idea that splitting of the first heart sound is due to asynchronism of ventricular contraction, and Katz (1925) has shown that slight asynchronism of ventricular contraction is present in the dog's heart. This finding has been confirmed in man by electrokymographic studies (Mednick et al., 1950), left ventricular 
ejection preceding right ventricular ejection in most instances (Hamilton et al., 1947; Ellinger et al., 1948).

Our observations have confirmed that there is normally some asynchronism of isometric ventricular contraction and of systolic ejection, and that the left precedes the right. They have also shown that, in the majority of subjects, the onset of left ventricular isometric contraction coincides with the first major component and that of right ventricular isometric contraction with the second major component of the first sound. It is generally believed that the auriculo-ventricular valves begin to close towards the end of auricular systole and that closure takes place simultaneously with the onset of isometric ventricular systole (Dean, 1916; Leatham and Vogelpoel, 1954; Braunwald et al., 1955). Therefore it seems reasonable to conclude that the first major component of the first sound is due to mitral valve closure and the second major component to tricuspid valve closure.

The observation that the amplitude of each component varies individually with its relationship to the time of onset of left and right auricular systole provides further supportive evidence for the suggestion that both components are due to similar events occurring on opposite sides of the heart. It would be difficult to account for the variation in amplitude of the second component relative to the time of auricular systole if one attributed its origin to semilunar valve opening. Moreover, aortic and pulmonary valve opening, timed by the electrokymogram, usually followed after the second major component. It is unlikely, therefore, that these events bear any relation to this sound. They were, however, related to the final component of the first heart sound the vibrations of which were best registered over the base of the heart and often continued for a short time after the onset of systolic ejection. It seems more probable that these vibrations are due to ejection of blood into the great vessels rather than to semilunar valve opening.

The second sound in young people is normally single on auscultation at the apex and second right intercostal space and split at the second left intercostal space. This also has been attributed to asynchronism of ventricular contraction. The first part, which is that heard at the apex and second right space, is due to aortic valve closure and it is believed that the second part is due to pulmonary valve closure (Abrahams and Wood, 1951; Leatham, 1952, 1954). Our observations support this belief by showing that the first part is closely related to the aortic dicrotic notch and the second to the pulmonary dicrotic notch on the electrokymogram. Increase in splitting of the second sound with inspiration is due to delayed closure of the pulmonary valve (Leatham and Towers, 1951) which in turn is probably due to overfilling of the right ventricle and prolongation of right ventricular systole.

The peak of the right auricular filling wave on the electrokymogram is known to correspond to the peak of the V wave on the right auricular pressure tracing (Rudhe, 1956), and it is therefore synchronous with tricuspid valve opening. There was a close relationship between the terminal vibrations of the second sound and tricuspid valve opening timed in this manner. Mitral valve opening, timed by the left auricular electrokymogram, appeared to occur after the final vibrations of the second sound in many instances. It is probable, however, that the terminal vibrations of the second sound were not always registered on the medium frequency phonocardiogram owing to attenuation of low frequencies in this channel. Therefore, it is concluded that the final vibrations of the second sound are probably related to opening of the auriculo-ventricular valves; but, because of their low amplitude and low frequencies in normal subjects, they are not audible on auscultation.

\section{CONCLUSIONS}

The first and second heart sounds are often audibly split in normal young people. Splitting of the first sound is most clearly heard at the lower left sternal border at the end of expiration, and of the second sound at the second left intercostal space at the height of inspiration.

The modern concept of the ætiology of the major components of the first and second heart sounds (Leatham, 1954) has been substantiated by their investigation with the aid of electrokymography. The two major components of the first sound that contribute to the splitting are separated 
by an average interval of $0.03 \mathrm{sec}$. and are of greater amplitude and contain more high-frequency vibrations than the other components. In most instances the first major component is synchronous with the onset of left ventricular isometric contraction and the second with the onset of right ventricular isometric contraction timed by the auricular electrokymogram. From this observation, it is concluded that the first major component is due to closure of the mitral valve and the second to closure of the tricuspid valve. Observations on the amplitude of each component relative to left and right auricular systole support this hypothesis. Semilunar valve opening, both aortic and pulmonary, occurs after the second major component in most cases; therefore, it is unlikely that this event has any relation to the audible components of the first heart sound.

Comparison of the aortic and pulmonary dicrotic notches with the two components of the split second sound has shown that the first is due to aortic valve closure and the second to pulmonary valve closure. They are separated by an average interval of $0.05 \mathrm{sec}$. in mid-respiration, but this increases during inspiration. Opening of the auriculo-ventricular valves does not contribute to the audible components of the second heart sound in normal people.

We are indebted to Dr. Philip Evans and Dr. Aubrey Leatham for their helpful criticism of this work.

\section{REFERENCES}

Abrahams, D. G., and Wood, P. H. (1951). Brit. Heart J., 13, 519.

Billing (1831-1832). Lancet, $2,198$.

Booth, E., Willis, K., Reeves, T. J., and Harrison, T. R. (1953). Circulation, 7, 916.

Bramwell, C. (1935). Quart. J. Med., 28 (4), 139.

Braunwald, E., Moscovitz, H. L., Amram, S. S., Lassa, R. P., Sapin, S. A., Himmelstein, A., Ravitch, M. M., and Gordon, A. J. (1955). Circulation, 12, 69.

Carswell (1832). Reported by Milne Edwards, $\dot{H}$. Leçons sur la physiologie et l'anatomie comparée de l'homme et des animaux faites à la Faculte des sciences de Paris. Paris V. Masson 1859, 4.

Cossio, P., and Lascales, M. (1936). Arch. Mal. Caur, 29, 138.

Counihan, T., Messer, A. L., Rappaport, M. B., and Sprague, H. B. (1951). Circulation, 3, 730.

Dean, A. L. (1916). Amer. J. Physiol., 40, 206.

Dock, W. (1933). Arch. intern Med., 51, 737.

Einthoven, W., and Geluk, M. A. J. (1894). Pfügers Arch. ges. Physiol., 57, 617.

Ellinger, G. F., Gillick, F. G., Boone, B. R., and Chamberlain, W. E. (1948). Amer. Heart J., 35, 971.

Geigel, R. (1906). Munch. med. Wschr., 53, 817.

Griffith, T. W. (1911-12). Heart, 3,143.

Hamilton, W. F., Attyah, A. M., Fowell, D. M., Remington, J. W., Wheeler, N. C., and Witham, A. C. (1947). Proc. Soc. Exp. Biol. Med., 65, 266.

Katz, L. N. (1925). Amer. J. Physiol., 72, 655.

Leatham, A. (1952). Brit. med. Bull., 8, 333.

(1954). Lancet, 2, 607.

二, and Towers, M. (1951). \begin{tabular}{c} 
Brit. Heart J., 13, 575. \\
\hline
\end{tabular}

Lewis, T. (1915). Lectures on the Heart. Paul B. Hoeber, New York.

Lian, C., Minot, G., and Welti, J. J. (1941). Phonocardiographie Auscultation Collective. Masson et cie, Paris.

Luisada, A. A. (1953). The Heart Beat. Paul B. Hoeber, New York.

, Mendoza, F., and Alimurung, M. M. (1949). Brit. Heart J., 11, 41.

Mannheimer, E. (1955). Advances in Pediatrics. Year book Publishers Inc. Vol. VII.

Mednick, H., Schwedel, J. B., and Samet, P. (1950). Circulation, 2, 250.

Nazzi, V., Ricco, G., and Meda, A. (1954). Cardiologia, 24, 319.

Orias, O., and Braun-Menendez, E. (1939). The Heart Sounds in Normal and Pathological Conditions. Oxford Univ. Press, London, New York, Toronto.

Potain, C. (1866). Bull. Soc. Med. Hôp., Paris, 3, 138.

Rappaport, M. B., and Sprague, H. B. (1942). Amer. Heart J., 23, 591.

Reinhold, J. D. L., Rudhe, U., and Bonham Carter, R. E. (1955). Brit. Heart J., 17, 327.

Rouanet, J. (1832). Analyse des Bruits du Cour. Thèse No. 252, Paris.

Rudhe, U. (1956). Acta Radiol. Suppl. 134.

Wiggers, C. J., and Dean, A. L. (1917). Amer. J. Physiol., 42, 476.

Wolferth, C. C., and Margolies, A. (1930). Arch. intern. Med., 46, 1048.

- - (1935). J. Clin. Invest., 14, 605.

- (1945). In Stroud's Diagnosis of Cardiovascular Disease. Vol 1. F. A. Davis Co., Phila. 